\title{
Exhaust of Underexpanded Jets from Finite Reservoirs
}

\author{
M. M. Orescanin, D. Prisco ${ }^{\dagger}$ and J. M. Austin ${ }^{\ddagger}$
}

\begin{abstract}
We examine the response of an underexpanded jet to a depleting, finite reservoir with experiments and simulations. An open-ended shock tube facility with variable reservoir length is used to obtain images of nitrogen and helium jet structures at successive instances during the blowdown from initial pressure ratios of up to 250 . The reservoir and ambient pressures are simultaneously measured to obtain the instantaneous pressure ratio. We estimate the time-scales for jet formation and reservoir depletion as a function of the specific heat ratio of the gas and the initial pressure ratio. The jet structure formation time-scale is found to become approximately independent of pressure ratio for ratios greater than 50. In the present work, no evidence of time-dependence in the Mach disk shock location is observed for rates of pressure decrease associated with isentropic blowdown of a finite reservoir while the pressure ratio is greater than 15 . The shock location in the finitereservoir jet can be calculated from an existing empirical fit to infinite-reservoir jet data evaluated at the instantaneous reservoir pressure. For pressure ratios below 15, however, the present data deviate from a compilation of data for infinite-reservoir jets. A new fit is obtained to data in the lower pressure regime. The self-similarity of the jet structure is quantified and departure from similarity is noted to begin at pressure ratios lower than about 15 , approximately the same ratio which limits existing empirical fits.
\end{abstract}

\section{Introduction}

The steady-state structure of a supersonic jet issuing from an infinite reservoir is well known to depend on the ratio between the reservoir and the ambient pressures. ${ }^{1,2}$ For an air jet issuing from an orifice with reservoir-to-ambient pressure ratio greater than about 5, the jet is underexpanded and a Mach disk shock exists at some distance from the vent. The Mach disk shock location can be theoretically predicted assuming the jet shear layer acts as a flow boundary and the pressure increase across the shock is required to match ambient conditions. ${ }^{3}$ At pressure ratios above 15, an empirical correlation to experimental data was reported by Ashkenas and Sherman and shown to be in good agreement with theory. ${ }^{4}$ The fit was independent of the specific heat ratio of the reservoir gas. Experiments were for jets exhausting from plenums with constant supply pressure over the test-time, simulating infinite reservoirs emitting steady-state jets.

There are many applications, however, where supersonic jets are created in the discharge from a finite reservoir, including small-scale propulsion and injection systems. For example, the jet structure and effectiveness of sonic fuel injection is studied across a broad range of applications and speed regimes. ${ }^{5-7}$ If the reservoir has finite length, the initial expansion fan will reflect from the end wall and propagate back towards the throat, decreasing the pressure of the reservior. The exhausting jet structure is consequently affected by the changing reservoir-to-ambient pressure ratio. In a spherical blast where the back pressure continually decreases, for example, experiments ${ }^{8}$ and analysis ${ }^{9}$ show the secondary shock initially propagates outwards, but then recedes back towards the origin. In a numerical study of open-ended shock tubes, Haselbacher et al. ${ }^{10}$ found that if the pressure ratio was sufficiently large that the expansion fan accelerated the flow to supersonic conditions, the exhausting expansion fan head affected the underexpanded jet structure. The propagation of the expansion wave reflected from a finite-reservoir end wall is complicated by interactions with the incident expansion, diffraction at the nozzle exit, and acceleration through and interaction with the jet exhaust, which may be turbulent. In the present work, we evaluate whether the jet response to a finite reservoir is unsteady or quasi-steady, as defined by the jet structure based on the Mach disk shock location measured in experiments.

\footnotetext{
* Graduate Student, University of Illinois

$\dagger$ Graduate Student, University of Illinois

¥Assistant Professor, Department of Aerospace Engineering, University of Illinois, Senior Member AIAA
} 
The initial establishment of the secondary shock structure may be also affected if the reservoir is finite. Upon sudden rupture of an overpressurized reservoir through an orifice, there is a transient evolution to the steady state structure of an underexpanded jet, as examined in detail by Radulescu and Law, Lacerda, and Naboko et al., among others. ${ }^{11-13}$ The initial shock and the jet head both propagate outwards and diffract due to the area change. A secondary shock system consisting of barrel and Mach disk shocks forms to match the pressure behind the diffracting shock wave with that of the expansion fan. The secondary shock structure initially forms in the vent corner region, then propagates behind an unsteady expansion head towards the vent centerline. The Mach disk shock forms at the centerline within a few vent diameters, and then propagates downstream towards the steady-state, or equilibrium, location.

The minimum times-scales required for supersonic jet establishment have been studied in the context of molecular beam experiments which are typically pulsed. ${ }^{14,15}$ A virtual hypersonic source model was developed by Chekmarev and Stankus ${ }^{16}$ and extended to analyze the far field by Radulescu and Law. ${ }^{11}$ In these models, the flow at the nozzle is instantaneously turned on and assumed to remain steady and constant over the time of formation, as would be the case for an infinite reservoir.

There is also recent evidence of flow history dependence of underexpanded jets after the secondary shock structure is established. A time-dependent reservoir-to-ambient pressure ratio has been previously shown to affect supersonic jet structure in the transition from regular to Mach reflection. ${ }^{17,18}$ Gribben et al. found hysteresis in the reflection transition of a Mach 3 jet using quasi-steady simulations. The Mach disk shock location as a function of decreasing or increasing pressure ratio was not reported. ${ }^{18}$ In a numerical study by Irie et al. ${ }^{17}$ the pressure ratio was varied by continuously increasing or decreasing the ambient pressure while the reservoir pressure remained constant. A pressure ratio range of 3 to 15 and pressure rate of changes of $\pm 1.167,0.835$, and 0.5 were selected. Their results show not only a hysteresis of the transition between Mach and regular reflection, but also a hysteresis in the location of the Mach disk shock location, decreasing below steady-state values for decreasing back pressure and increasing above for increasing back pressure. The critical pressure for Mach to regular reflection transition was found to depend on the flow history and a hysteresis loop was calculated. ${ }^{17}$ The damping effect of non-equilibrium condensation on hysteresis was examined by Kim et al. ${ }^{19}$ Pressure ratios of 3.0 to 6.2 were examined in increments of 0.1 in simulations and experiments. Both dry and moist jets exhibited hysteresis, with Mach disk shock location over-predicted for increasing pressure ratio and under predicted for decreasing pressure ratio. Moist jets from square and equilateral triangular nozzles were examined in experiments by Umeda et al. ${ }^{20}$ Hysteresis phenomenon were not however observed in jets from these vent geometries. These previous studies focused on the transition from regular to Mach reflection in the jet structure.

In the present work, we instead examine the response of the Mach disk shock location to a changing pressure ratio due to reservoir depletion. The reservoir pressure monotonically decreases at a rate that is a function of time. The reservoir is depleted by reflected expansion waves which also exit the reservoir and interact with the jet exhaust in a complex manner. We address the following outstanding questions for the startup and blowdown of a supersonic exhaust from a finite reservoir: Can time-scales associated with jet establishment and reservoir depletion be estimated? In a "small" finite reservoir, is the formation of a Mach disk shock interrupted by reflected waves? Once established, can the jet structure be predicted from steady-state theory if the reservoir pressure is known, or does the flow history play a role?

Our goal is to evaluate whether the finite-reservoir jet is unsteady or quasi-steady as defined by the Mach disk shock structure (although other definitions of unsteady are of course possible). A series of experiments and simulations focused on understanding the effect of decreasing reservoir pressure on jet structure are carried out. Time-resolved images are obtained at successive intervals during the blowdown. Data for a range of initial pressure ratios up to 250 and reservoir volume to exit area ratios from 1 to 26 times the diameter of the nozzle are examined. Results are compared with existing studies of underexpanded jets from infinite reservoirs. The paper is organized as follows: The experimental setup for the laboratory and numerical experiments are described in Section II.A. Time-resolved images of jet structure during the discharge and pressure histories in the reservoir are presented in Section III. The jet depletion and Mach disk shock formation time-scales are estimated and compared in Section IV.A. This estimate neglects the external diffraction and complex propagation of the reflected characteristics through the jet exhaust. Experiments are then used to examine the jet response to reflected waves from reservoir of different length in Section IV.B. Shock structure measurements for jets exiting depleting reservoirs are compared to existing data for infinite reservoirs. 


\section{Experimental and Numerical Setups}

\section{II.A. Experimental Configuration}

Experiments were conducted in an open-ended shock tube facility, designed to access initial pressure ratios up to 250, and a range of reservoir dimensions with nitrogen and helium test gases. A reservoir containing test gas initially pressurized up to $4.5 \mathrm{MPa}$ exhausts either into the ambient atmosphere or into a vacuumcapable test section, Figure 1. The reservoir is a constant $55 \mathrm{~mm}$ diameter pipe in which cylindrical blockages can be inserted to change the reservoir length in stages from $38 \mathrm{~mm}$ to $965 \mathrm{~mm}$, varying the volume while maintaining similar one-dimensional internal gas dynamics. Mounted to the discharging end of the reservoir is one of two convergent nozzles with throat diameters $\mathrm{D}$ of $10 \mathrm{~mm}$ or $4 \mathrm{~mm}$. A Mylar diaphragm is initially located at the nozzle throat, separating the high pressure reservoir from the downstream test region.

Pressure ratios up to 250 are achieved by combining the reservoir with a test section evacuated down to $10 \mathrm{kPa}$ minimum pressure (Figure 1). The optically-accessible test section is large enough to act as an unconfined volume and to ensure a negligible back pressure increase during the experiment. The test section includes a $4 \mathrm{~m}$ pipe extension that allows the turbulent downstream jet wake to propagate without interference and that increases the total volume. A static pressure transducer (Setra 206) with a $5 \mathrm{~ms}$ response time measures the initial reservoir pressure. Dynamic pressure transducers (PCB 113A26) with $1 \mu \mathrm{s}$ response times measure the pressure history both in the reservoir and in the test section during the jet exhaust. More experimental detail can be found in Orescanin. ${ }^{21}$

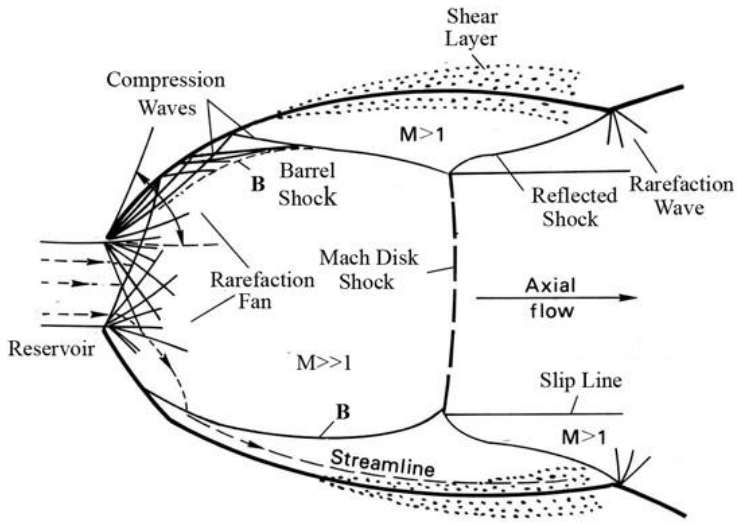

a)

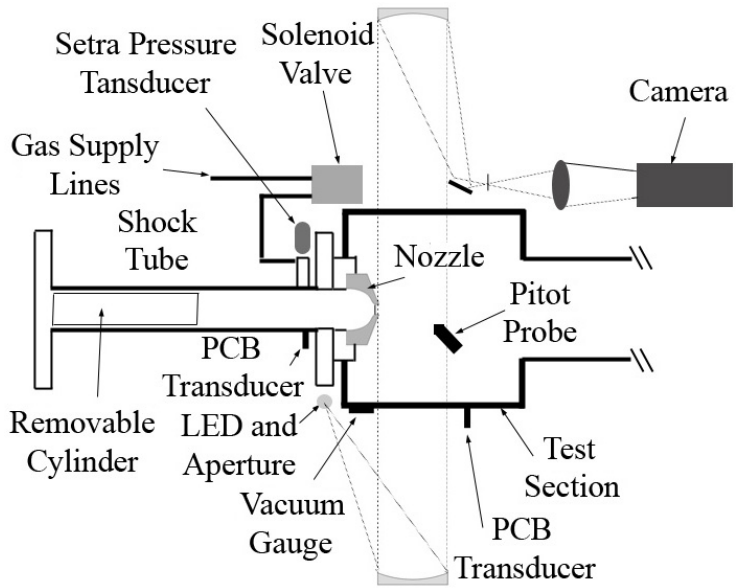

b)

Figure 1. a) Schematic showing shock structure of an underexpanded jet. ${ }^{22}$ b) Schematic of experimental setup (not to scale) of the open-ended shock tube facility. The diaphragm is located at the nozzle throat. The reservoir length is adjusted by the inserting solid cylinders of different length. The schlieren setup is used to obtain images of the jet during reservoir discharge while the transducers record the initial burst pressure (Setra transducer), and the pressure profiles in the reservoir and test section (PCB transducers). The arrival of the initial blast wave at the pitot probe triggers all data acquisition.

Data acquisition is triggered by the arrival of the initial shock wave at a sting-mounted dynamic pressure gauge (PCB 113A26) located in the free field, off-axis from the jet exhaust. Single-shot schlieren images of the jet structure at selected times are obtained for each experiment. Light from a continuous white-light LED point source is collimated to a $102 \mathrm{~mm}$ field of view and images are recorded with a pco.1600 CCD camera (Cooke Corporation) with $100 \mu$ s exposure time at delays from 0 to $100 \mathrm{~ms}$ after diaphragm rupture preset using a Quantum Composer delay generator. Data acquisition with a single time origin allows schlieren images to be compared with the reservoir pressure history to yield the instantaneous pressure ratio for each image, and visualization of the discharging jet structure from each initial condition is constructed from a sequence of successive single-shot experiments. Experiments are conducted with four reservoir lengths $L$ : $38 \mathrm{~mm}, 152 \mathrm{~mm}, 228 \mathrm{~mm}$, and $965 \mathrm{~mm}$, with resulting reservoir ratios $\frac{V_{r}}{A_{n} D}$ of $1.0,8.5,10$, and 26 respectively, where $V_{r}$ is the reservoir volume and $A_{n}$ is the nozzle area. 


\section{II.A.1. Error analysis}

The error in experimental determination of Mach disk shock location due to both measurement precision and image scaling was $\pm 0.4 \mathrm{~mm}$. Gauges used to measure the pressure history in the reservoir during each experiment had $1 \mu$ s response time. During this time, the pressure decreased by less than $2 \%$. Error bars for the experimental data are generated based on these values and are included in Section III.

\section{II.B. Numerical Simulations}

The initial stages of jet formation are investigated with axisymmetric numerical simulations. The nonreacting Euler equations are solved with the Amrita environment using an operator-split scheme with HLLE flux and kappa-MUSCL reconstruction. An adaptive mesh refinement (AMR) algorithm is incorporated. The computational scheme is documented in Quirk. ${ }^{23,24}$ Amrita computations are carried out on a 450 by 630 coarse grid, with 60 cells across the vent radius. Two levels of additional refinement and a refinement factor of three are incorporated for the AMR. The refinement criteria are based on density gradients to locate shocks and on a local comparison between density and pressure gradients to locate contact surfaces. ${ }^{24}$ A CFL number of 0.6 is used. To determine that the Mach disk shock structure formation time (the main result for this study from the simulations) is not dependent on the computational domain, a simulation with a factor of two increase in the coarse grid is carried out. The uncertainty in the formation time $t_{f}$, non-dimensionalized by the reservoir sound speed $c_{4}$ and diameter $D$, is chosen by the time-step between data output, and was $\pm 0.01 \frac{c_{4} t_{f}}{D}$.

The initial condition consists of a contact surface separating the high pressure reservoir and the ambient surroundings. A constant specific heat ratio was assumed with $\gamma=1.4$. Extrapolation boundary conditions were applied to simulate an unconfined volume into which the jet exhausts. The reservoir centerline is assumed to be an axis of symmetry.

\section{Experimental Measurements of Mach Disk Shock Location}

Temporal histories of the jet as the reservoir pressure decreases were constructed from single-shot schlieren images acquired at selected times after diaphragm rupture, Figure 2. The first image is obtained at the time of incident blast-wave arrival at the pitot probe, at $0.2 \mathrm{~ms}$ after diaphragm rupture. In all experiments we see the Mach disk shock fully developed at its equilibrium location by the time the camera is triggered. As the reservoir pressure decreases as a function of time, the Mach disk shock propagates back towards the vent and decreases in diameter (Figures 2b, c, d, and e). The barrel shocks contract into the flow and the overall area of the supersonic region decreases through the blowdown. Between successive images taken at pressure ratios of 6.5 and 4.2, there is a transition from Mach reflection to regular reflection, and a series of oblique shock waves appear in the exhaust, replacing the Mach disk shock. The Mach disk disappears altogether as the reservoir pressure decreases past this threshold (Figure 2f). This transition is consistent with the pressure ratio of 5 previously reported. ${ }^{17}$ Since the focus of the present work is the propagation of the Mach disk shock in response to changing reservoir pressure during the blowdown, no further effort was made to identify the transition pressure ratio more exactly. As the reservoir pressure continues to decrease beyond that shown in Figure $2 \mathrm{f}$ and Figure 3d, the nozzle throat becomes unchoked and the flow is everywhere subsonic.

Schlieren images of helium jet structure shortly after the diaphragm rupture are shown in Figure 3. Images are shown for reservoir lengths of $96.5 \mathrm{~cm}$ to $3.8 \mathrm{~cm}$. The initial reservoir pressure was 40, and the instantaneous pressure ratio at the time of image acquisition is reported. A significantly more turbulent jet shear layer is observed in the case of helium, however barrel and Mach disk shock structures still can be identified.

The reservoir pressure for each image was obtained by measurement of the time history of pressure within the reservoir. The pressure history is determined by the initial reservoir volume, the nozzle exit area, the speed of sound of the gas, $c_{4}$, and the ratio, $R_{p}$, of instantaneous reservoir pressure, $P_{4}(t)$, to the initial reservoir pressure, $P_{4}(0)$. Assuming an isentropic process, the reservoir pressure ratio $R_{p}$ as a function of time is ${ }^{25}$

$$
R_{p}(t)=\frac{P_{4}(t)}{P_{4}(0)}=\left(\frac{t c_{4}}{V_{r} / A_{n}}\left[\left(\frac{\gamma-1}{2}\right)\left(\frac{2}{\gamma+1}\right)^{\frac{\gamma+1}{2(\gamma-1)}}\right]+1\right)^{\frac{2 \gamma}{1-\gamma}}
$$




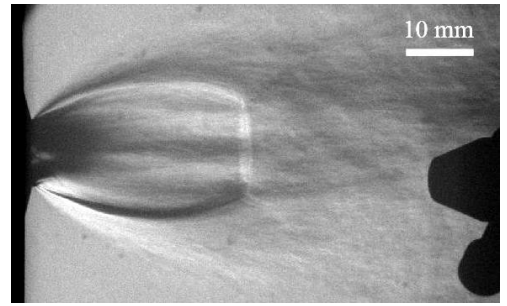

a) $0.2 \mathrm{~ms}$

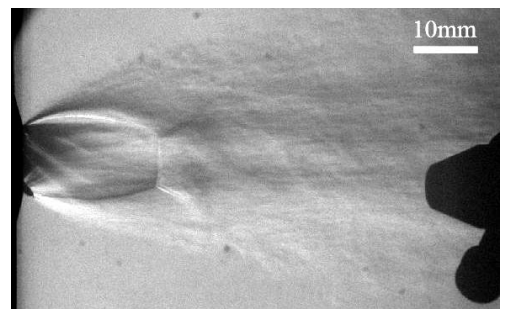

d) $5 \mathrm{~ms}$

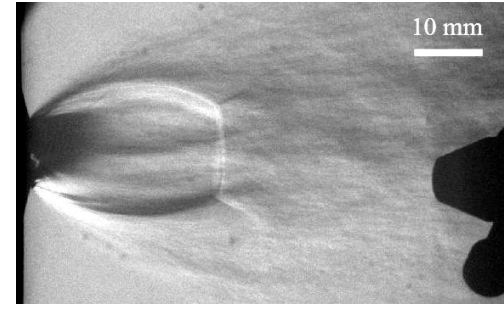

b) $1 \mathrm{~ms}$

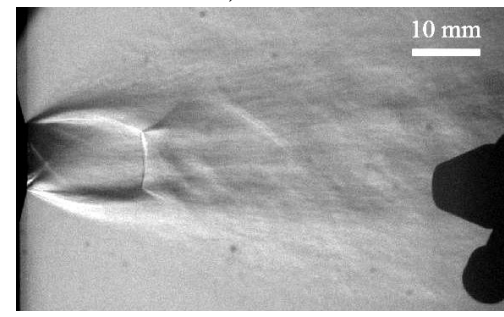

e) $7 \mathrm{~ms}$

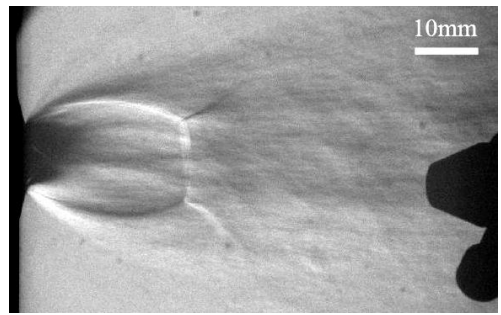

c) $2 \mathrm{~ms}$

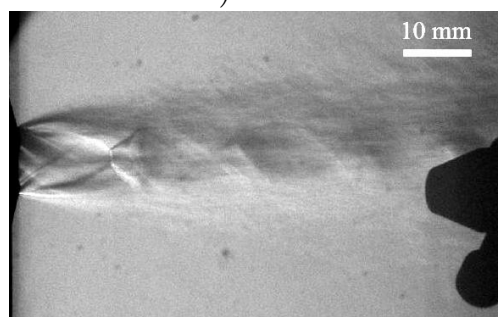

f) $10 \mathrm{~ms}$

Figure 2. Schlieren images of a nitrogen jet exhausting from a $38 \mathrm{~mm}$ long reservoir into ambient air. Flow is from left to right. The reservoir was filled with nitrogen at initial pressure ratio of a) 40:1, with subsequent instantaneous pressure ratios of: b) $28: 1$, c) $17: 1$, d) $13: 1$, e) $8: 1$ and f) $6: 1$. Image acquisition is triggered by the arrival of the blast wave at a pressure gauge, evident on the right side of each frame as the black object, and times shown are relative to diaphragm burst.

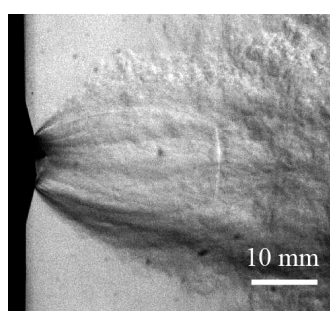

a) $5 \mathrm{~ms}$

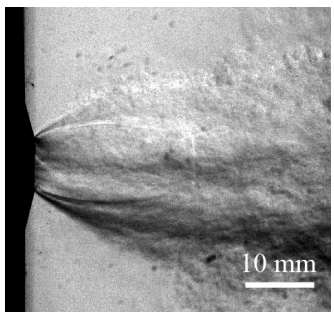

b) $5 \mathrm{~ms}$

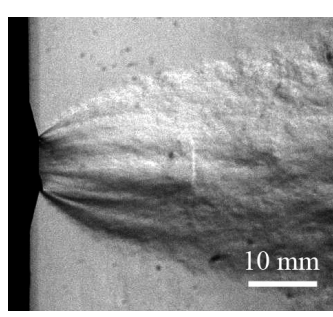

c) $5 \mathrm{~ms}$

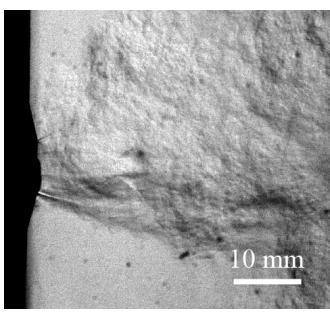

d) $2 \mathrm{~ms}$

Figure 3. Schlieren images of the helium jet structure exhausting into ambient air. Initial reservoir pressure was 40 . a) instantaneous pressure ratios of 35, reservoir length $96.5 \mathrm{~cm}$; b) instantaneous pressure ratios of 26 , reservoir length $19.1 \mathrm{~cm}$; c) instantaneous pressure ratios of 25, reservoir length $11.4 \mathrm{~cm}$; and d) instantaneous pressure ratios of 9, reservoir length $3.8 \mathrm{~cm}$. Image acquisition is triggered by the arrival of the blast wave at a pressure gauge, evident as the black object on the right side of each frame, and times are shown relative to the diaphragm burst. 
The reservoir depletion rate can be obtained by differentiating this expression. Experimental nondimensional plots for pressure and time rate of change of pressure are compared to Equation 1 in Figure 4. In nondimensional coordinates, the experimental pressure histories collapse on to the single curve given by Equation 1, Figure 4a.

Hysteresis has been reported in the literature for the location of the Mach disk shock, ${ }^{17}$ as discussed in Section I. Irie et al. considered constant pressure derivatives of $\pm 1.167,0.835$, and 0.50 . In order to compare the results of the present study, the nondimensional time derivative for the pressure ratio decay is calculated from a fit to the experimental data and from the theoretical curve given by Equation 1, and shown in Figure 4b. The nondimensional time rate of change of the pressure discharge for the universal, isentropic blowdown curve is comparable in the initial stages to values examined by Irie et al. ${ }^{17}$

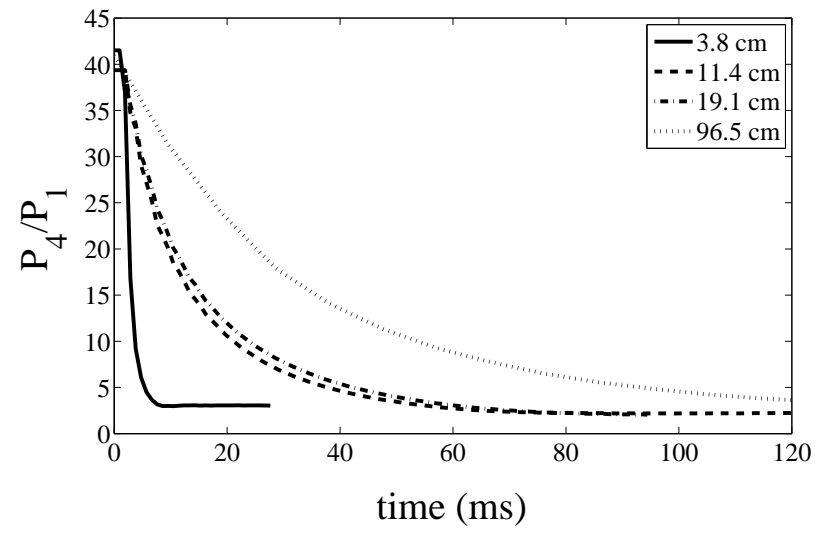

a)

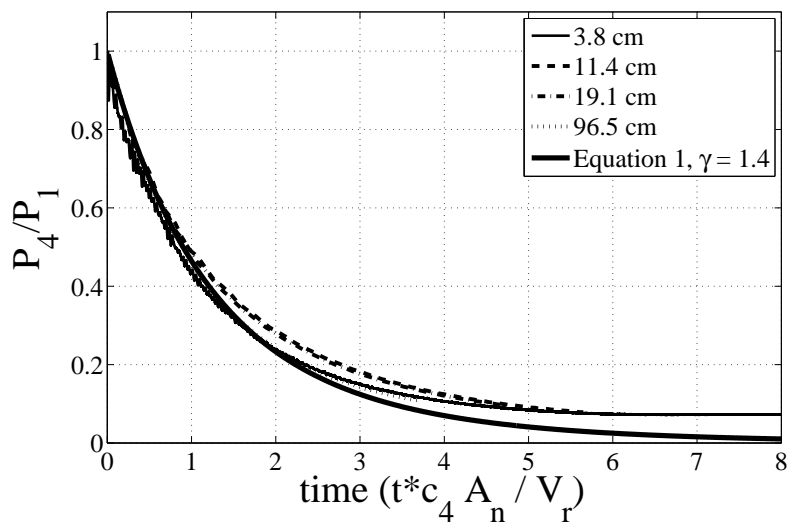

c)

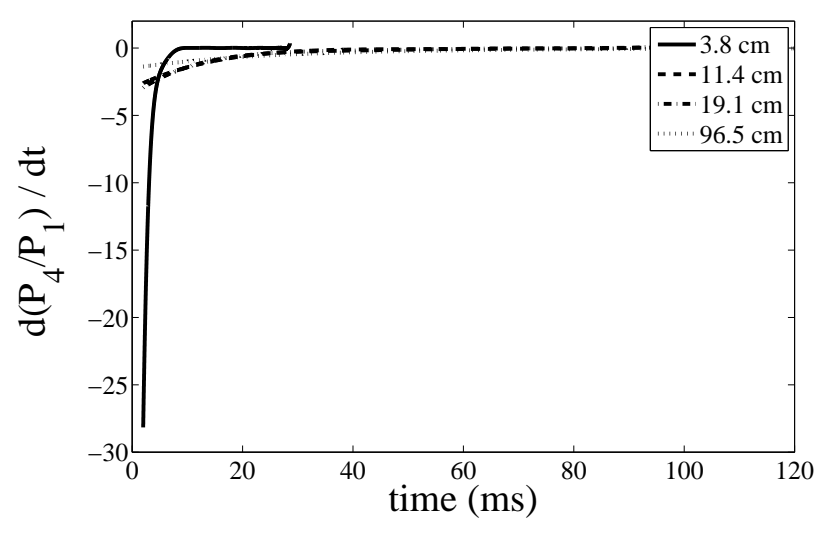

b)

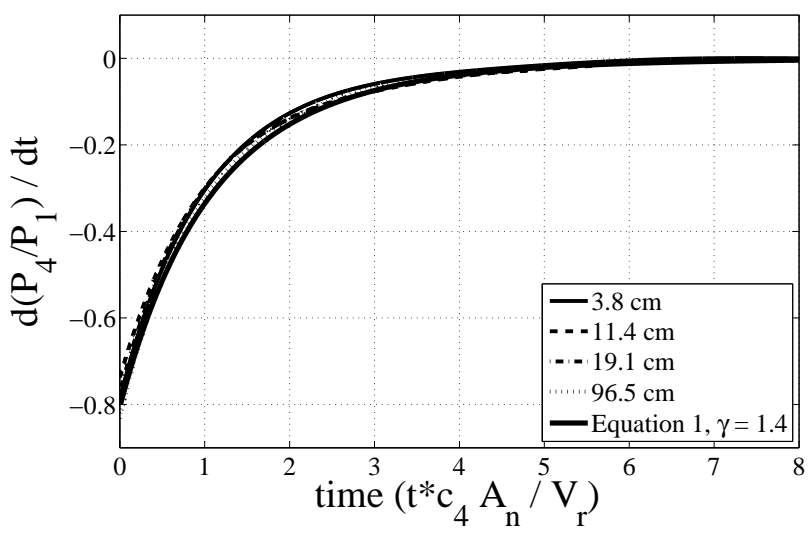

d)

Figure 4. Sample dimensional a) reservoir pressure histories and b) pressure derivatives for the discharge of a helium jet reservoirs of different lengths with initial pressure ratio of 40 . Nondimensionalized c) pressure and d) pressure derivatives from a fit to the experimental data and from the theoretical curve given by Equation 1 which represents the nondimensional scaling for an isentropic process.

\section{Results and Discussion}

\section{IV.A. Time-scales of Jet Startup and Reservoir Blowdown}

The transient process of underexpanded jet structure formation have been identified for steady-state nozzles, for example Radulescu and Law. ${ }^{11}$ We examine the conditions under which the the discharge timescale from a short reservoir is comparable to the Mach disk shock formation time. The timescales of reservoir blowdown are estimated from one-dimensional gas dynamic calculations. The timescales of Mach disk shock formation are estimated by examining the shock diffraction process, theoretically and from numerical simulations. We compare the range of time scales for jets of different $\gamma$, initial pressure ratio, and reservoir dimensions. 


\section{IV.A.1. Reservoir Depletion Time-Scales}

The pressure in a finite reservoir during isentropic discharge decreases due to passage of reflected expansion waves. We estimate the initial two-way travel time for an expansion wave in the reservoirs of different lengths used in the experiments. The reservoir is assumed to be a straight duct and nozzle contraction is neglected in this analysis. The exit plane is assumed choked, corresponding to initial pressure ratios above 10 and 4 for nitrogen and helium jets respectively from one-dimensional shock tube theory.

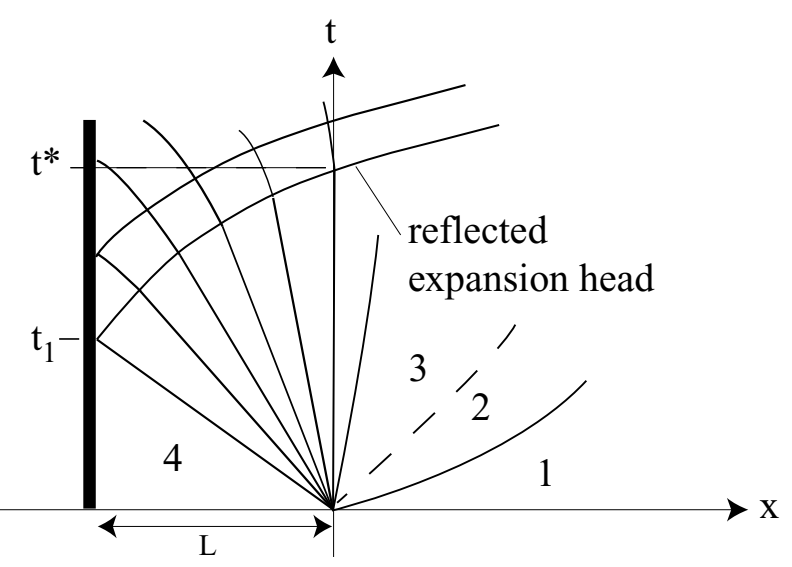

Figure 5. Nomenclature for the calculation of the arrival time of the reflected expansion head at the vent exit $x=0$. The one-dimensional calculation applies in the reservoir region $x<0$. (The flow field for $x>0$ is external to the reservoir and multi-dimensional.)

The time of arrival of the first characteristic of the reflected expansion head (and the boundary of the simple region) can be obtained via a similarity solution. ${ }^{26}$ This solution accounts for the interaction of the reflected head with the incident wave, Figure 5 . The similarity variable $\eta=\frac{x}{c_{4} t}$ satisfies an ordinary differential equation derived from the method of characteristics through a left-facing expansion fan centered at $x=0$

$$
t \frac{d \eta}{d t}+2 \eta \frac{\gamma-1}{\gamma+1}-\frac{4}{\gamma+1}=0
$$

where $\gamma$ and $c_{4}$ are the specific heat ratio and sound speed respectively of the reservoir gas. The equation can be integrated to obtain an implicit relationship for time of the reflected expansion head arrival at the vent $\mathrm{t}^{*}$

$$
\frac{\gamma+1}{2}{\frac{L}{c_{4} t^{*}}}^{\frac{2(\gamma-1)}{\gamma+1}}+\frac{L}{c_{4} t^{*}}-\frac{2}{\gamma-1}=0
$$

where the reservoir has length $\mathrm{L}$. Calculated times based on this analysis are shown in Table 1. For the shorter reservoir length, the two-way travel time of waves inside the reservoir is less than the schlieren image exposure, for the two intermediate reservoirs, it is comparable, and for the longest reservoir, the resolution of the experiments is a factor of three to four faster than the travel time of a reflected characteristic.

\section{IV.A.2. Jet Formation Time-Scales}

An analogous flowfield to transient jet startup occurs when a shock wave propagating in a duct encounters an abrupt area change and undergoes a multi-dimensional diffraction. In an experimental study, Skews ${ }^{27}$ obtained schlieren images that identified the features of the flow. An unsteady expansion fan is generated at the corner. The head of the expansion fan propagates along the incident shock, causing it to diffract. For strong shocks with supersonic outflow, the unsteady expansion head is convected downstream. A steady Prandtl-Meyer expansion fan exists at the corner. The pressure and velocity decrease through the expansion fan is greater than the decrease behind the diffracting shock wave. ${ }^{9}$ As a result, a secondary (Mach disk) shock system is formed, initially offset from the vent exit in the corner region of the steady expansion. This secondary shock system is the origin of the first Mach disk and barrel shocks in an underexpanded jet and 
Table 1. Two-way travel time, $t_{1}+t^{*}$, calculations for the reflected expansion head to reach the throat for conditions of the experiments, where $t_{1}$ is the time of arrival of the expansion head at the end wall, Figure 5. One-dimensional gas dynamic calculations account for the interaction of the incident and reflected expansion fans, but neglect the nozzle contraction.

\begin{tabular}{|c|c|c|c|c|}
\hline Reservoir $V_{r} / A_{n} D$ & $\begin{array}{c}\text { Nitrogen } \\
(\mu \mathrm{s})\end{array}$ & $\begin{array}{c}\text { Helium } \\
(\mu \mathrm{s})\end{array}$ & $\begin{array}{c}\text { Nitrogen } \\
\frac{c_{4}\left(t_{1}+t^{*}\right)}{D}\end{array}$ & $\begin{array}{c}\text { Helium } \\
\frac{c_{4}\left(t_{1}+t^{*}\right)}{D}\end{array}$ \\
\hline 1.0 & 35 & 27 & 12 & 27 \\
8.5 & 138 & 107 & 47 & 109 \\
10 & 207 & 261 & 71 & 163 \\
26 & 877 & 680 & 300 & 691 \\
\hline
\end{tabular}

it propagates towards the vent centerline following an unsteady expansion. Other flow features which were observed include a shear layer generated due to boundary layer separation, and a contact surface which separates gas processed by the lead shock from gas processed by the secondary shock.

Numerical simulations are used to obtain an estimate of the time-scales required for the Mach disk shock to reach the tube centerline, Figure 6 . Simulations were performed for an air/air interface with pressure ratios of 40,150, and 250. The nondimensional time for the Mach disk shock to reach the tube centerline was measured to be $\frac{t_{f} c_{4}}{D}=1.3$ for all three cases, corresponding to $t_{f}=0.04 \mathrm{~ms}$ for the present experiments. No appreciable dependence of formation time on pressure ratio was observed over the range of pressure ratios considered.

Comparison of formation times with reflected expansion wave travel times shows that even for reservoirs with $V_{r} / A_{n}$ of about one diameter, the formation time of the Mach disk shock is an order of magnitude less than the travel time of the reflected expansion head to the nozzle exit plane. In addition, the Mach disk shock forms within a few diameters of the vent, and the diffraction and propagation of the reflected expansion to this location is neglected. Thus even for a constant-area, finite reservoir with length over diameter ratios of one, the Mach disk shock has ample time to form.

A first estimate of the conditions under which the formation of the Mach disk structure may be affected by the reflected expansion head may be obtained by setting the two-way travel time of the first characteristic inside the reservoir equal to the formation time obtained in simulations:

$$
\frac{c_{4}\left(t_{1}+t *\right)}{D}=1.3
$$

Substituting this into Equation 3 for $t^{*}$ gives a relationship between the reservoir length and diameter as a function of $\gamma$ :

$$
\frac{\gamma+1}{2} \frac{L}{1.3 D-L}^{\frac{2(\gamma-1)}{\gamma+1}}+\frac{L}{1.3 D-L}-\frac{2}{\gamma-1}=0
$$

This estimate of the critical condition at which time-scales of reflected head arrival at vent exit and Mach disk shock formation at the vent centerline are comparable is shown in Figure 7. In reservoirs with L/D ratios below approximately one, the expansion fan arrival at the nozzle exit occurs before the normal shock formation at the exit centerline.

The analysis above neglects the propagation of the reflected expansion head through the jet structure, where the wave propagation is complicated by diffraction at the area change and interaction with flowfield of the jet exhaust. The response of the shock structure may not be instantaneous to the arrival of the first reflected characteristic as assumed. In addition, after initially forming in the vicinity of the throat, the Mach disk shock structure itself evolves and propagates downstream towards its equilibrium location. The authors are only aware of one study that examined the propagation of the Mach disk to its steady state location in detail. ${ }^{12}$ Lacerda observed that the flow remained unsteady for substantially longer than the shock arrival time as the shock was observed to exhibit a damped oscillation about the equilibrium location. We therefore use our experimental results to examine the response of the jet after shock formation to a depleting reservoir. 


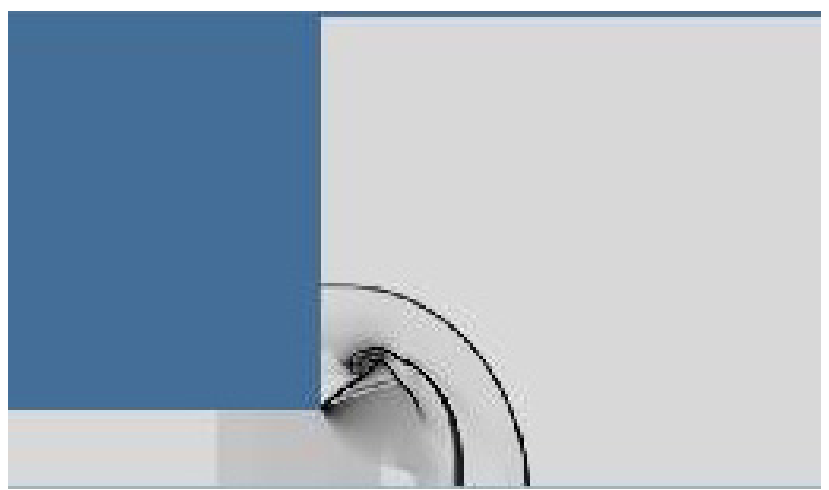

a) $\mathrm{t}_{4} / \mathrm{D}=0.64$

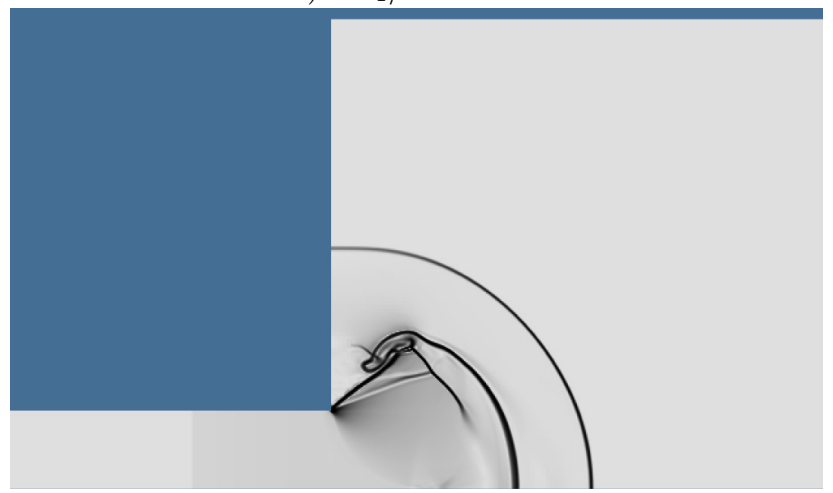

b) $\mathrm{t} \mathrm{c}_{4} / \mathrm{D}=0.86$

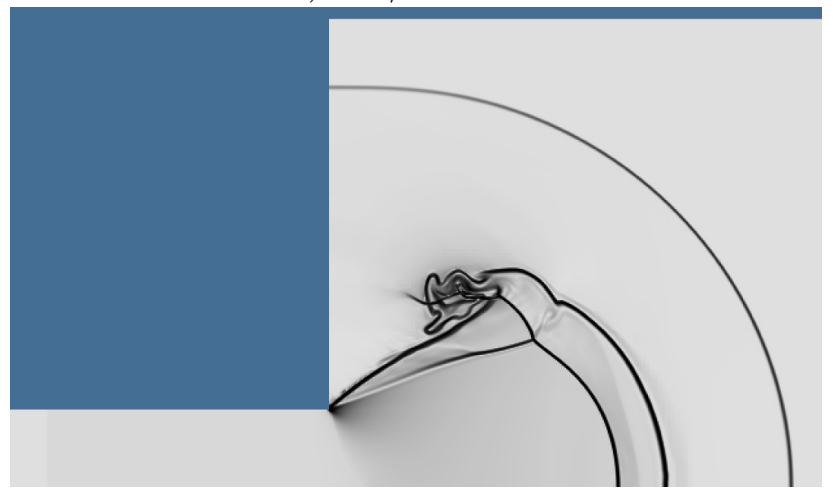

c) $\mathrm{t}_{4} / \mathrm{D}=1.78$

Figure 6. Formation of Mach disk shock in an nitrogen jet from a reservoir with pressure ratio of 40. The calculation is axisymmetric about the reservoir centerline. 


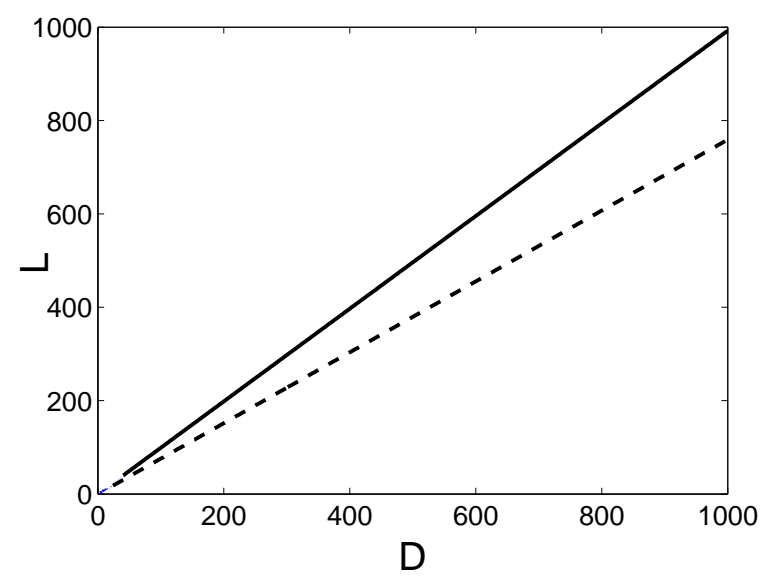

Figure 7. Estimate of the critical vent dimensions for which time-scales of reflected head arrival at vent exit and Mach disk shock formation at the vent centerline are comparable, for $\gamma=1.4$ (solid line) and $\gamma=1.67$ (dashed line). For L/D ratios below the plotted line, the expansion fan arrival at the nozzle exit occurs before the normal shock formation at the exit centerline.

\section{IV.B. Evaluation of Quasi-Steady Hypothesis for Discharge from a Finite Reservoir}

We examine whether the dynamic shock structure observed in experiments during the reservoir blowdown can be predicted assuming a quasi-steady flow. That is, if the reservoir pressure is instantaneously known, can the Mach disk shock location be calculated from steady-state results?

In an underexpanded jet from an infinite reservoir, the non-dimensional location of the first Mach disk shock normalized by the nozzle diameter depends on the reservoir to ambient pressure ratio. The method of characteristics, ${ }^{28}$ or an approximate solution, ${ }^{3}$ can be used to calculate the location of the shear layer which is assumed to act as a flow boundary. As in a nozzle expansion, the Mach disk shock location can be predicted assuming the pressure increase across the shock is required to match ambient conditions. Experimental data show the Mach disk shock location normalized by the exit diameter is linearly dependent on the square root of the reservoir-to-ambient pressure ratio for pressures greater than $15 .^{2,4} \mathrm{~A}$ compilation of theoretical results and experimental data fits for infinite-reservoir jets is shown in Figure 8. A power-law empirical fit for pressure ratios between 15 and 10000 was derived from continuous-flow facility experimental data by Ashkenas and Sherman: ${ }^{4}$

$$
\frac{x_{m}}{D}=0.67\left(\frac{P_{4}}{P_{1}}\right)^{0.5}
$$

Theoretical results are in good agreement with compiled experimental data for pressure ratios greater than 15, Figure 8.

The location of Mach disk shock relative to the vent at selected times after diaphragm rupture is measured from experimental data. Reservoir pressures at these times are known from the experimental traces. The normalized Mach disk shock locations are shown in Figures 9 and 10 for nitrogen and helium jets respectively. The difference in initial burst pressures creates no observable difference in Mach disk shock location as a function of pressure ratio. For each initial burst pressure, the distance falls initially on the empirical curve at the correlating maximum pressure, then propagates along this curve as the pressure ratio between the reservoir and downstream conditions decreases. The Mach disk locations for pressure ratios above 15 agree with the infinite-reservoir jet locations given by the Ashkenas-Sherman relation, Equation 6. Agreement is the same for both nitrogen and helium, indicating this result is independent of the ratio of specific heats. There is no dependence on the initial pressure ratio, nor on the reservoir length.

To form a basis for comparison with our finite-reservoir data outside the pressure range valid for the Ashkenas and Sherman fit, we compile available experimental and numerical data from the literature for pressure ratios lower than 15 for infinite-reservoir jets, Figure 11. There is considerably less agreement between theory and experiment at lower pressure ratios, as pointed out by Ashkenas and Sherman. ${ }^{4}$ The Adamson and Nicholls model predicts a decrease in the shock location due to a nonlinear decrease in the centerline pressure close to the vent. ${ }^{3}$ Experiments also show a decreased Mach disk shock location, however 


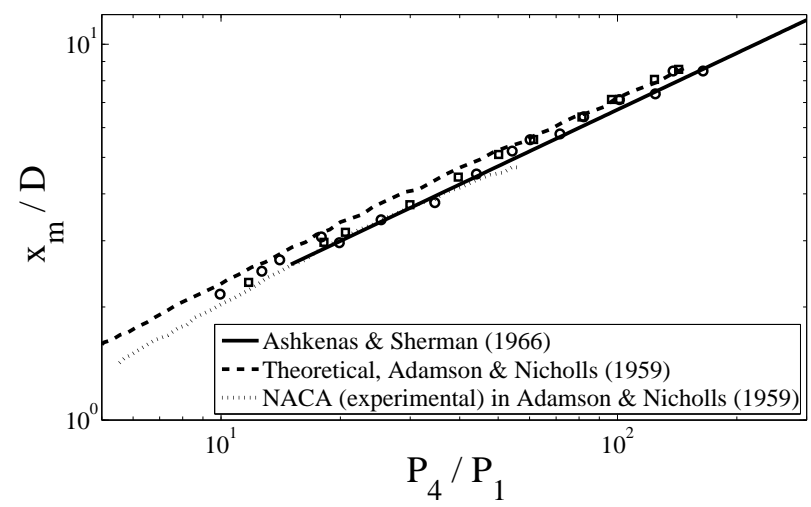

Figure 8. Normalized Mach disk shock location, $x_{m} / D$, versus reservoir-to-ambient pressure ratio, $P_{4} / P_{1}$. Experimental data are from APL $(\square)$, as reported in Adamson and Nicholls, ${ }^{3}$ and Crist et al. $(o) .{ }^{2}$ The Ashkenas and Sherman fit is valid for pressure ratios greater than 15. ${ }^{4}$ The extrapolation of the Ashkenas and Sherman to lower pressures is shown as a heavy dashed line. Data and theory are for an underexpanded jet with constant supply pressure. The fits were found to be independent of $\gamma$.

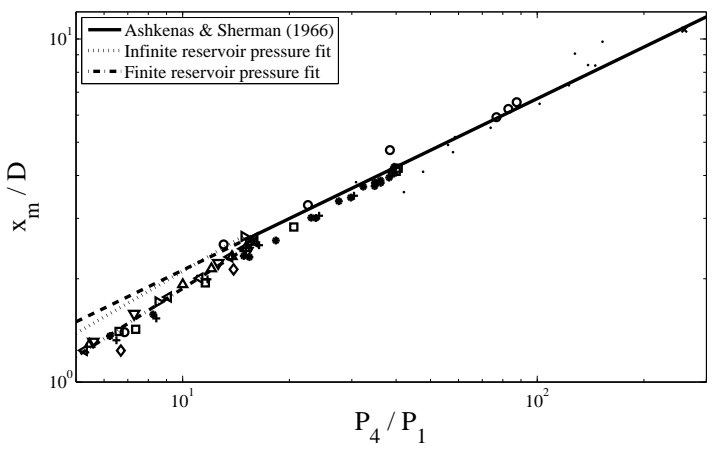

Figure 9. Mach disk shock location, $x_{m} / D$, versus reservoir to ambient pressure ratio, $P_{4} / P_{1}$ for nitrogen jets from finite reservoirs. Initial reservoir-to-ambient pressure ratios were $250(\times), 150(\bullet), 100(\circ),, 40(\square,+, *, \diamond)$, and $15(\nabla, \triangle, \triangleleft, \triangleright)$. Reservoir lengths of $96.5 \mathrm{~cm}(\square, \nabla), 22.8 \mathrm{~cm}(+, \triangle), \mathbf{1 5 . 2} \mathrm{cm}(\circ, \bullet, \times)$, and $3.8 \mathrm{~cm}(\diamond, \triangleright)$. The error in the pressure measurement is less than the symbol size. The extrapolation of the Ashkenas and Sherman for to lower pressures is shown as a heavy dashed line.

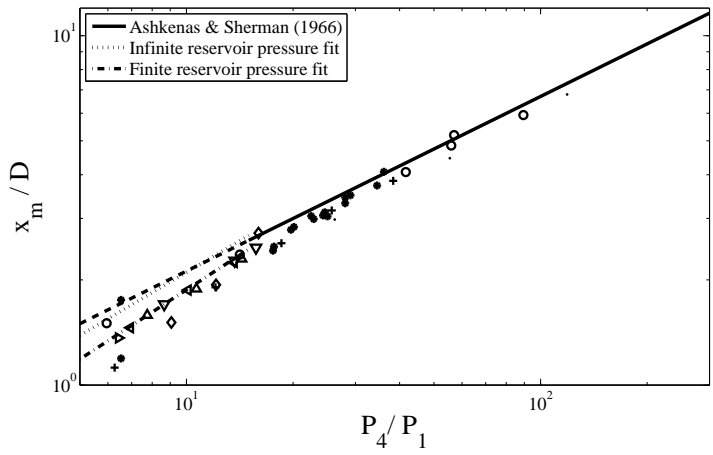

Figure 10. Mach disk shock location, $x_{m} / D$, versus reservoir to ambient pressure ratio, $P_{4} / P_{1}$ for helium jets from finite reservoirs. Initial reservoir-to-ambient pressure ratios of $150(\bullet), 100(\circ),, 40(\square,+, *, \diamond)$, and $15(\nabla, \triangle, \triangleleft, \triangleright)$. Reservoir lengths of $96.5 \mathrm{~cm}(\square, \nabla), 22.8 \mathrm{~cm}(+, \triangle), 15.2 \mathrm{~cm}(\circ, \bullet, \times)$, and $3.8 \mathrm{~cm}(\diamond, \triangleright)$. The error in the pressure measurement is less than the symbol size. 


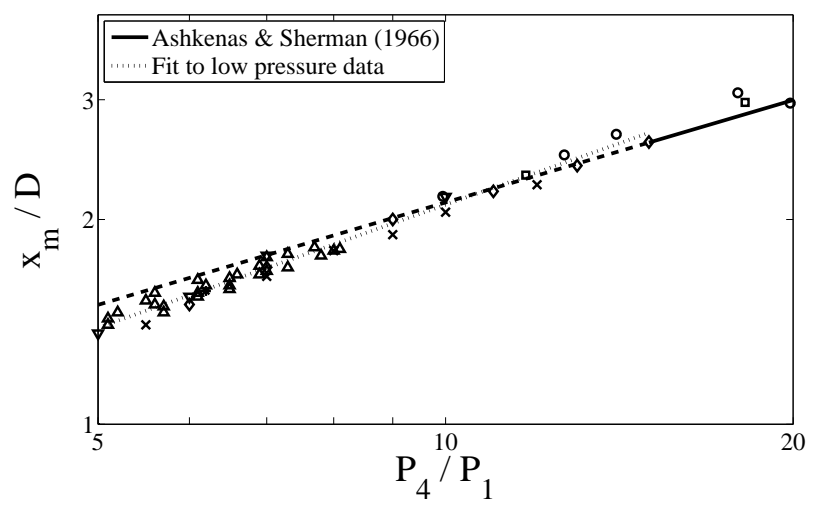

Figure 11. Normalized Mach disk shock location, $x_{m} / D$, versus reservoir-to-ambient pressure ratio, $P_{4} / P_{1}$, in the low pressure regime. Data are from the experiments of Crist et al. $(o),{ }^{2} \mathrm{Kim}$ et al.( $(\bullet),{ }^{19}$ Otobe et al. $(\times),{ }^{29} \mathrm{Baek}$ et al. $(\triangle),{ }^{30}$ and the simulations of Birkby et al. $(\nabla),{ }^{31}$ and Irie et al. $(\diamond) \cdot{ }^{17}$ All data are for underexpanded jets with constant supply pressure. An empirical fit to the compilation of literature results for pressure ratios up to 15 is shown. The extrapolation of the Ashkenas and Sherman for to lower pressures is shown as a heavy dashed line.

the shock appears significantly closer to the vent than predicted by theory, Figure 11. We obtain an empirical curve fit to the compilation of infinite-reservoir jet data from the literature, shown in Figure 11:

$$
\frac{x_{m}}{D}=0.53\left(\frac{P_{4}}{P_{1}}\right)^{0.6}
$$

The data from present blowdown experiments are compared to this fit and to an extrapolation of the Ashkenas and Sherman fit to lower pressures in Figure 9. Error bars are evaluated as discussed in Section II.A. Below a pressure ratio of 15 , the data systematically fall below the fits derived for infinite-reservoir jets. These observations are again independent of reservoir length and initial pressure ratio and hold for both nitrogen and helium test gases, however, there is a greater spread in the data for helium below a 15 pressure ratio.

We calculate a fit to the present data sets for pressure ratios less than 15 :

$$
\frac{x_{m}}{D}=0.41\left(\frac{P_{4}}{P_{1}}\right)^{0.66}
$$

The same fit is obtained for both nitrogen and helium jets.

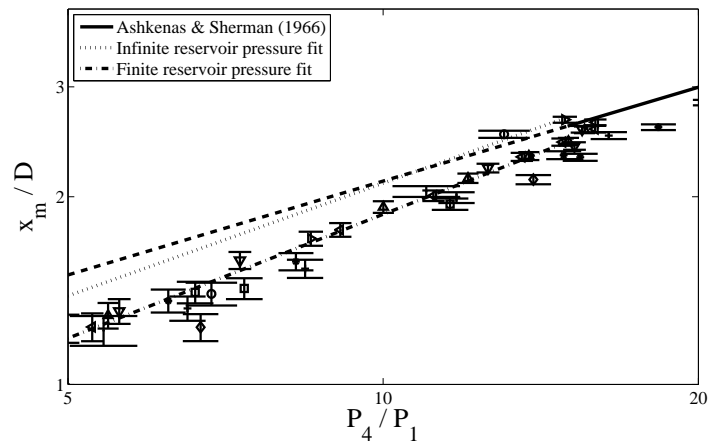

Figure 12. Mach disk shock location, $x_{m} / D$, versus reservoir to ambient pressure ratio, $P_{4} / P_{1}$ for nitrogen jets from finite reservoirs, in the low pressure regime. Initial reservoir-to-ambient pressure ratios were $250(\times)$, $150(\bullet)$, $100(\circ$,$) ,$ $40(\square,+, *, \diamond)$, and $15(\nabla, \triangle, \triangleleft, \triangleright)$. Reservoir lengths of $96.5 \mathrm{~cm}(\square, \nabla), 22.8 \mathrm{~cm}(+, \triangle), 15.2 \mathrm{~cm}(\circ, \bullet, \times)$, and $3.8 \mathrm{~cm}(\diamond, \triangleright)$. The error in the pressure measurement is less than the symbol size.

Irie et al. examined three different rates of change of the pressure $\frac{d\left(P_{4} / P_{1}\right)}{d t}= \pm 1.167,0.835$, and 0.5 , where the last two cases are comparable to the initial stages of isentropic blowdown. The Mach disk shock was reported to be located further downstream than the equilibrium location if the pressure ratio was decreasing. 


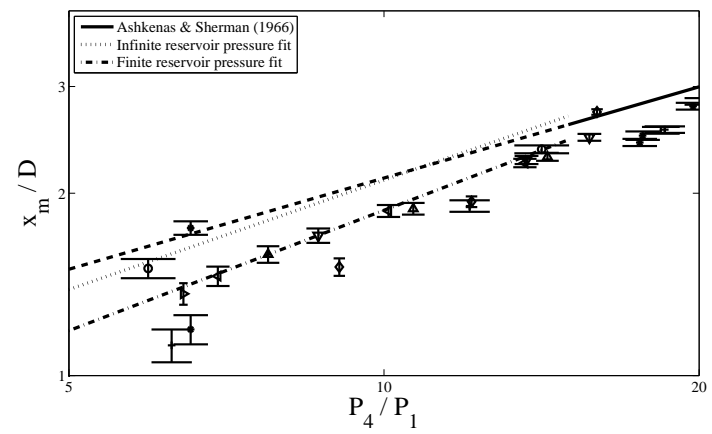

Figure 13. Mach disk shock location, $x_{m} / D$, versus reservoir to ambient pressure ratio, $P_{4} / P_{1}$ for helium jets from finite reservoirs, in the low pressure regime. Initial reservoir-to-ambient pressure ratios of $150(\bullet), 100(\circ),, 40(\square,+, *, \diamond)$, and $15(\nabla, \triangle, \triangleleft, \triangleright)$. Reservoir lengths of $96.5 \mathrm{~cm}(\square, \nabla), 22.8 \mathrm{~cm}(+, \triangle), 15.2 \mathrm{~cm}(\circ, \bullet, \times)$, and $3.8 \mathrm{~cm}(\diamond, \triangleright)$. The error in the pressure measurement is less than the symbol size.

In the present work, we instead find that the Mach disk shock location is over predicted by steady-state theory when the pressure ratio is less than 15 .

In summary, the experimental data show the reservoir history has no effect on the location of the Mach disk shock through the blowdown of the jet to pressure ratios greater than 15 . This result is independent of the initial reservoir pressure, of $\gamma$, and of the reservoir length. Instead, the shock location follows the Ashkenas and Sherman relation for infinite-reservoir jets, Equation 6, evaluated at the instantaneous reservoir pressure. For pressure ratios greater than 15, time-dependence is not observed even at the initial stages of the blowdown where the pressure ratio derivative is greatest. Below a pressure ratio of 15 , however, the location of the Mach disk shock in both infinite- and finite-reservoir jets deviates from the Ashkenas and Sherman fit. Equation 7 represents the authors' fit to the compilation of existing infinite-reservoir jet data from the literature in this pressure range. There is considerably more scatter in the data in the lower pressure ratio regime, however, within this uncertainty, the present data are appreciably different to infinite-reservoir results.

The self-similarity of underexpanded jet structure at large pressure ratios has been demonstrated by Falle, but as the focus of this work was extragalatic jets, the near-vent region and low pressure jets were not considered. ${ }^{32}$ We examine the possible self-similarity of the secondary shock structure for varying pressure ratios in the depleting jet. Barrel and Mach disk shock features were traced from schlieren images at pressure ratios of 6 to 150, scaled, and overlaid. A compilation of contours from selected images is shown in Figure 14a. Selected scaling factors for different pressure ratios are shown in Table 2. Overlays were compiled for i) images obtained at a constant delay from reservoirs of different initial pressure ratio, and ii) successive images during a fixed blowdown condition. In both cases, the jet structure is found to be self-similar while the pressure ratio remains above about 15 . Over the range of pressure ratios from about 15 to 12 , the shock structure departs gradually but noticeably from self-similarity, losing curvature in the barrel shock structure. We note this to coincide with the pressure ratio where the Ashkenas and Sherman fit no longer describes the Mach disk standoff distance.

As a means of quantifying the degree of the departure, the ratio of the width of the Mach disk shock $y_{m}$ to the maximum width of the barrel shock $y_{B}$ is measured from the images at different pressure ratios, Figure 14b. For higher pressure ratios, $\frac{y_{m}}{y_{B}}$ is approximately constant. When the pressure ratio during the blowdown reaches approximately 12, the width of the Mach disk shock decreases abruptly relative to the maximum barrel width. The Adamson and Nicholls model predicts a decrease in the shock location due to a nonlinear decrease in the reservoir and nozzle exit pressures close to the vent. ${ }^{3}$ Love and Grigsby found the extent of the boundary between the orifice and the maximum jet diameter can be fairly well approximated by a circular arc. ${ }^{1}$ Their calculations show the arc radius is approximately constant for jet exit to ambient pressure ratios greater than about 20, then decreases over the pressure range of 20 to 4 . This is consistent with the current observations that the structure can no longer be mapped to a self-similar profile at lower pressures. 


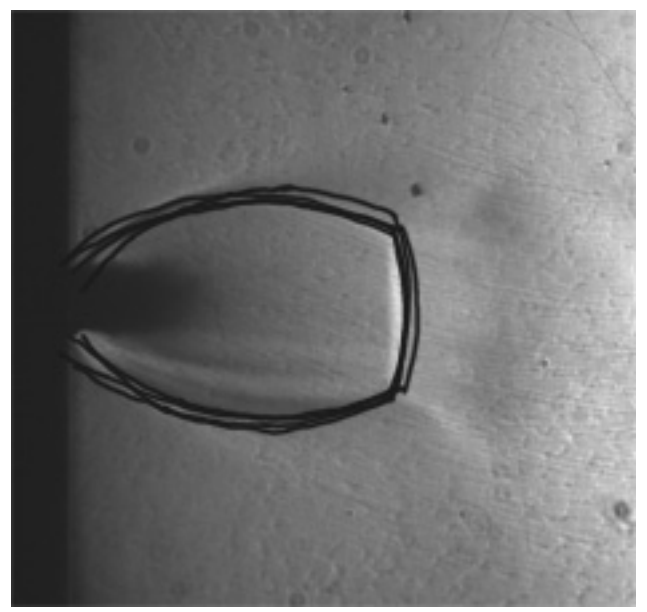

a)

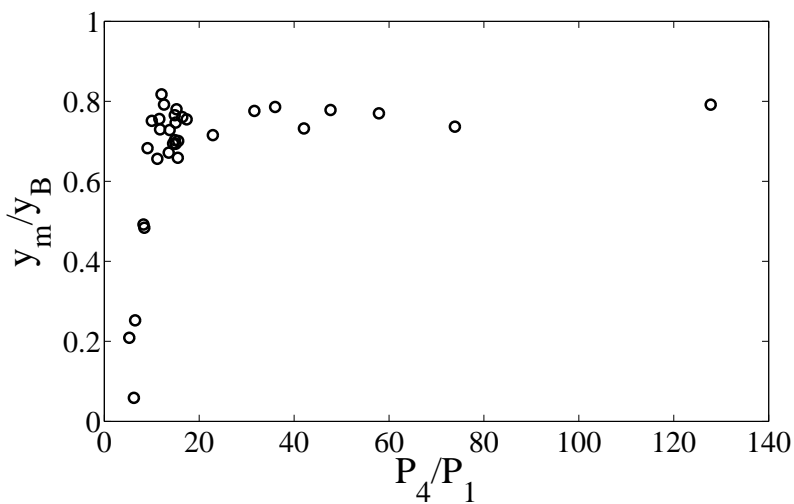

b)

Figure 14. a) Overlay of scaled Mach disk and barrel shock structures traced from schlieren images. Initial pressure ratios from 127.8 to 14.5 , Table 2. b) Measured ratios of the maximum width of the barrel shock $y_{B}$ to the width of the Mach disk shock $y_{m}$ at different pressure ratios.

Table 2. Scaling factors applied to overlay barrel and Mach disk shock structures for selected values of the instantaneous pressure ratio.

\begin{tabular}{|c|cccccc|}
\hline Pressure ratio & 127.8 & 73.9 & 35.6 & 22.9 & 17.4 & 14.5 \\
\hline Scaling factor & 61.9 & 100 & 149 & 187 & 221 & 246 \\
\hline
\end{tabular}




\section{Conclusions}

The Mach disk shock structure of an underexpanded jet issuing from an infinite reservoir is known to depend on the reservoir-to-ambient pressure ratio. In the current study, we examined the response of the Mach disk shock location to a (non-constant) decreasing pressure ratio associated with the isentropic discharge of a finite reservoir. We posed the following questions: Can time-scales associated with jet establishment and reservoir depletion be estimated? In a "small" finite reservoir is the formation of a Mach disk shock interrupted by reflected waves? Once established, can the jet structure be predicted from steady-state theory if the reservoir pressure is known, or does the flow history play a role?

As an indication of the rate of reservoir depletion, the timescales for the propagation of reflected expansion waves in the reservoir were calculated using the method of characteristics, neglecting the nozzle area contraction. The interaction of the reflected head with the initial wave was modeled using a similarity solution. The non-dimensional time-of-arrival of the reflected head at the nozzle throat was found to vary from $\frac{c_{4}\left(t_{1}+t^{*}\right)}{D}=12$ to 300 in nitrogen and $\frac{c_{4}\left(t_{1}+t^{*}\right)}{D}=27$ to 691 in helium, for reservoir volume to area ratios from 1 to 26 diameters.

The formation time of the Mach disk shock was estimated from axially symmetric numerical simulations using the Amrita environment. Simulations indicate the formation time becomes independent of pressure ratio at ratios greater than about 50. Formation times for nitrogen jets from reservoirs with initial pressure ratios from 40 to 250 were $\frac{t_{f} c_{4}}{D}=1.3$, an order of magnitude less than the time-of arrival of the reflected expansion head, even for a shortest $\left(\frac{V_{r}}{A_{n} D}=1.0\right)$ reservoir considered. An estimate of the critical vent dimensions for which time-scales of reflected head arrival at vent exit and Mach disk shock formation at the vent centerline are comparable was made.

Once the reflected expansion wave exits the nozzle, it diffracts and interacts with the jet exhaust. The response of the underexpanded jet was examined experimentally in an open shock tube facility with variable reservoir length. The experimental configuration was chosen such that the reservoir volume could be varied while similar internal gas-dynamics during the discharge were maintained. Schlieren images of nitrogen and helium jet structure were obtained at selected intervals during the blowdown while the reservoir pressure history was measured.

The location of the Mach disk shock at successive instances was compared with existing results for jets with constant supply pressure. While the pressure ratio remained above 15, the Mach disk shock location could be predicted based on infinite-reservoir jet results if the instantaneous pressure ratio was known. That is the Mach disk shock location during the depleting discharge followed the Ashkenas and Sherman empirical fit for infinite-reservoir jets.

For pressure ratios less than 15, the Ashkenas and Sherman relation is no longer valid, and a curve was fit to the compilation of existing infinite-reservoir jet data from the literature. The Mach disk shock location in the finite-reservoir jet is systematically less than that of the infinite-reservoir jet, both of which are less than the extrapolation of the higher pressure Ashkenas and Sherman fit. The undershoot in the Mach disk shock location in the present experiments is in contrast with the simulations of Irie et al. who examined hysteresis in the regular to Mach reflection location by changing the back pressure. Irie et al. found the Mach disk shock location was over-predicted if the back pressure was increasing. The results in the present experiments were independent of $\gamma$, reservoir dimensions, or the initial reservoir pressure ratio. The independence of initial pressure ratio and therefore of the magnitude of the pressure derivative at the time of departure from infinite-reservoir results indicates the instantaneous pressure ratio is the dominant parameter at these conditions. In contrast, Irie et al. found increasing the pressure derivative increased the undershoot in Mach disk shock location. The rates of pressure decay in the present study are associated with an isentropic discharge, and except for the initial stage of the blowdown, are substantially more modest than those prescribed by Irie et al.

The jet structure was found to be self-similar at pressure ratios above about 15 . The departure from selfsimilarity was quantified by a decrease in the Mach disk shock width to the maximum barrel width at pressure ratio between 12 and 15 . The pressure ratio for departure from self-similarity was found approximately to correspond to a change in the jet boundary radius of curvature as calculated by the method of characteristics.

Thus for the conditions of this study, the Mach disk shock structure will form within a few diameters of the nozzle, unaffected by interaction with the reflected expansion fan associated with the finite reservoir. Once the Mach disk shock has propagated downstream and is established, the location of the shock in depleting reservoir jets can be predicted from steady-state theory while the reservoir pressure remains above 
15. Below this pressure ratio, there is a departure from infinite-reservoir results which also approximately coincides with a departure from self-similarity.

\section{Acknowledgments}

The authors gratefully acknowledge interactions with Prof. Susan Kieffer in this study. This work was supported in part by NSF grant EAR06-09712, NSF grant SK2008-0035 8 ANTC and Charles R. Walgreen endowed funds to Susan W. Kieffer. We are grateful to Dr. James Quirk for the use of his code Amrita. We thank David Buchta for performing modeling runs with a different code to cross-check our results.

\section{References}

${ }^{1}$ Love, E. and Grigsby, C., "Some Studies of Axisymmetric Free Jets Exhausting from Sonic and Supersonic Nozzles into Still Air and into Supersonic Streams," Tech. Rep. RM L54L31, NACA, 1955.

${ }^{2}$ Crist, S., Sherman, P., and Glass, D., "Study of the Highly Underexpanded Sonic Jet," AIAA Journal, Vol. 4, No. 1, 1966, pp. 68-71.

${ }^{3}$ Adamson, T. and Nicholls, J., "On The Structure of Jets From Highly Underexpanded Nozzles Into Still Air," Journal of Aeronautical Sciences, Vol. 26, No. 1, 1959, pp. 16-24.

${ }^{4}$ Ashkenas, H. and Sherman, F. S., "Experimental Methods in Rarefied Gas Dynamics," Tech. Rep. 32-869, NASA, 1966.

${ }^{5}$ Ben-Yakar, A., Mungal, M. G., and Hanson, R., "Time Evolution and Mixing Characteristics of Hydrogen and Ethylene Transverse Jets in Supersonic Crossflows," Physics of Fluids, Vol. 18, No. 2, 2006, pp. 026101, doi:10.1063/1.2139684.

${ }^{6}$ Viti, V., Wallis, S., Schetz, J. A., Neel, R., and Bowersox, R. D. W., "Jet Interaction with a Primary Jet and an Array of Smaller Jets," AIAA Journal, Vol. 42, No. 7, 2004, pp. 1358-1368, doi:10.2514/1.4850.

${ }^{7}$ Alvi, F., Shih, C., Elavarasan, R., and Krothapalli, A., "Control of Supersonic Impinging Jet Flows Using Supersonic Microjets," AIAA Journal, Vol. 41, No. 7, 2003, pp. 1347-1355, doi:10.2514/2.2080.

${ }^{8}$ Boyer, D., "An Experimental Study of the Explosion Generated by a Pressurized Sphere," Journal of Fluid Mechanics, Vol. 9, 1960, pp. 401, doi:10.1017/S0022112060001195.

${ }^{9}$ Friedman, M., "A Simplified Analysis of Spherical and Cylindrical Blast Waves," Journal of Fluid Mechanics, Vol. 11, 1961, pp. 1-15, doi:10.1017/S0022112061000810.

${ }^{10}$ Haselbacher, A., Balachandar, S., and Kieffer, S., "Open-Ended Shock Tube Flows: Influence of Pressure Ratio and Diaphragm Position," AIAA Journal, Vol. 45, No. 8, 2007, pp. 1917-1929, doi:10.2514/1.23081.

${ }^{11}$ Radulescu, M. I. and Law, C., "The Transient Start of Supersonic Jets," Journal of Fluid Mechanics, Vol. 578, 2007, pp. 331-369, doi:10.1017/S0022112007004715.

${ }^{12}$ Lacerda, N., On the Start Up of Supersonic Underexpanded Jets, Ph.D. thesis, Graduate Aeronatical Laboratories, California Institute of Technology, Pasadena, CA, 1987.

${ }^{13}$ Naboko, I., Belavin, V., and Golub, V., "Nonstationary Wave Structure of Intermittent Supersonic Jet," Acta Astronautica, Vol. 6, 1979, pp. 885-890.

${ }^{14}$ Saenger, K., "Pulsed Molecular Beams: A Lower Limit on Pulse Duration for Fully Developed Supersonic Expansions," Journal of Chemical Physics, Vol. 75, No. 5, 1981, pp. 2467-2469, doi:10.1063/1.442272.

${ }^{15}$ Korobeishchikov, N., Zarvin, A., and Madirbaev, V. Z., "Hydrodynamics of Pulsed Supersonic Underexpanded Jets: Spatiotemporal Characteristics," Technical Physics, Vol. 49, No. 8, 2004, pp. 973-981, doi:10.1134/1.1787654.

${ }^{16}$ Chekmarev, K. and Stankus, N., "Gas-Dynamic Models and Relationship of Similarities for Launchings of Hypersonic Nozzles and Jets," Zhurnal Tekhnichesko Fiziki, Vol. 54, No. 8, 1984, pp. 1576.

${ }^{17}$ Irie, T., Yasunobu, T., Kashimura, H., and Setoguchi, T., "Characteristics of a Mach Disk in the Underexpanded Jet in Which the Back Pressure Continuously Changes with Time," Journal of Thermal Sciences, Vol. 12, No. 2, 2003 , pp. 132-137.

${ }^{18}$ Gribben, B., Badcock, K., and Richards, B., "Numerical Study of Shock-Reflection Hysteresis in an Underexpanded Jet," AIAA Journal, Vol. 38, No. 2, 2000, pp. 275-283, doi:10.2514/2.954.

${ }^{19}$ Kim, H.-D., Kang, M.-S., Otobe, Y., and Setoguchi, T., "The Effect of Nonequilibrium Condensation on Hysteresis Phenomenon of Underexpanded Jets," Journal of Mechanical Science and Technology, Vol. 23, No. 2, 2009, pp. 856-867, doi:10.1007/s12206-009-0207-9.

${ }^{20}$ Umeda, Y. and Ishii, R., "Oscillation Modes of Underexpanded Jets Issuing from Square and Equilateral Triangular Nozzles," J. Acoustic Soc. Am., Vol. 95, No. 4, 1994, pp. 1853-1857, doi:10.1121/1.408700.

${ }^{21}$ Orescanin, M. S. M., An Experimental Study of the Dynamics Governing Supersonic Volcanic Jets: Mount St. Helens, May 18, 1980, Master's thesis, Department of Geology, University of Illinois, Urbana, IL, 2009.

${ }^{22}$ Kieffer, S., "Fluid Dynamics of the May 18 Blast at Mount St. Helens," U.S. Geological Survey Professional Paper 1250, edited by P. Lipman and D. Mullineaux, 1982, pp. 379-400.

${ }^{23}$ Quirk, J., "Amrita: A Computational Facility for CFD Modeling," 29th Computational Fluid Dynamics Lecture Series, ed. H. Deconinck, von Karman Institute, 1998, http://www.amrita-cfd.org/doc.

${ }^{24}$ Quirk, J., "Amr_sol: Design Principles and Practice," 29th Computational Fluid Dynamics Lecture Series, ed. H. Deconinck, von Karman Institute, 1998, http://www.amrita-cfd.org/doc.

${ }^{25}$ Kieffer, S. and Sturtevant, B., "Laboratory Studies of Volcanic Jets," Journal of Geophysical Research, Vol. 89, No. B10, 1984, pp. 8253-8268, doi:10.1029/JB089iB10p08253.

${ }^{26}$ Wintenberger, E., Austin, J. M., Cooper, M., Jackson, S. I., and Shepherd, J. E., "An Analytical Model for the Impulse of Single-Cycle Pulse Detonation Tube," AIAA Journal of Propulsion and Power, Vol. 19, No. 1, 2003, pp. 22-38. 
${ }^{27}$ Skews, B., "The Perturbed Region Behind a Diffracting Shock Wave," Journal of Fluid Mechanics, Vol. 29, 1967, pp. 705-719, doi:10.1017/S0022112067001132.

${ }^{28}$ Love, E., "An Approximation of the Boundary of a Supersonic Axisymmetric Jet Exhausting into a Supersonic Stream," Journal of Aeronautical Sciences, Reader's Forum, Vol. 25, No. 2, 1958, pp. 130-131.

${ }^{29}$ Otobe, Y., Kashimura, H., Matsuo, S., Setoguchi, T., and Kim, H., "Influence of Nozzle Geometry on the NearField Structure of a Highly Underexpanded Sonic Jet," Journal of Fluids and Structures, Vol. 24, No. 2, 2008, pp. 281-293, doi:10.1016/j.jfluidstructs.2007.07.003.

${ }^{30}$ Baek, S., Kwon, S., Kim, H., Setoguchi, T., and Matsuo, S., "Study of Moderately Underexpanded Supersonic Moist Air Jets," AIAA Journal, Vol. 44, No. 7, 2006, pp. 1624-1627, doi:10.2514/1.10029.

${ }^{31}$ Birkby, P. and Page, G., "Numerical Predictions of Turbulent Underexpanded Sonic Jets Using a Pressure-Based Methodology," Proceedings of the Society of Mechanical Engineers, Part G, Journal of Aerospace Engineering, Vol. 215, No. 3, 2001, pp. 165-173, doi:10.1243/0954410011533158.

${ }^{32}$ Falle, S. A. E. G., "Self-Similar Jets," Monthly Notices of the Royal Astronomical Society, Vol. 250, 1991, pp. 581-596. 